Mens

revue d'histoire intellectuelle de l'Amérique française

\title{
Incursion personnaliste chez les thomistes canadiens-français des années 1930 et 1940 : l'exemple de François Hertel
}

\section{Marie Martin-Hubbard}

Volume 6, numéro 1, automne 2005

URI : https://id.erudit.org/iderudit/1024255ar

DOI : https://doi.org/10.7202/1024255ar

Aller au sommaire du numéro

Éditeur(s)

Centre de recherche en civilisation canadienne-française

ISSN

1492-8647 (imprimé)

1927-9299 (numérique)

Découvrir la revue

Citer cet article

Martin-Hubbard, M. (2005). Incursion personnaliste chez les thomistes canadiens-français des années 1930 et 1940 : l'exemple de François Hertel. Mens, 6(1), 29-67. https://doi.org/10.7202/1024255ar
Résumé de l'article

François Hertel, à l'image des élites canadiennes-françaises de l'époque, est thomiste dans les années trente. La restauration de la doctrine philosophique et religieuse de Thomas d'Aquin a profondément marqué le catholicisme québécois; mais en dépit des apparences, le thomisme n'est pas monolithique au Canada français dans l'entre-deux-guerres. Des différences d'interprétation de Thomas d'Aquin engendrent des conceptions de l'homme et de sa fin appelées à s'opposer. Hertel, par son discours même, incarne ces tensions; malgré sa facture thomiste, Pour un ordre personnaliste (1942), le deuxième essai de l'écrivain, embrasse un personnalisme qui incite les uns et les autres à se prononcer sur le bien-fondé d'une distinction entre l'individu et la personne. 


\title{
INCURSION PERSONNALISTE CHEZ LES THOMISTES CANADIENS-FRANÇAIS DES ANNÉES 1930 ET 1940 : L'EXEMPLE DE FRANÇOIS HERTEL
}

\author{
Marie Martin-Hubbard \\ Département des lettres françaises \\ Université d'Ottawa
}

\section{Résumé}

François Hertel, à l'image des élites canadiennes-françaises de l'époque, est thomiste dans les années trente. La restauration de la doctrine philosophique et religieuse de Thomas d'Aquin a profondément marqué le catholicisme québécois ; mais en dépit des apparences, le thomisme n'est pas monolithique au Canada français dans l'entre-deux-guerres. Des différences d'interprétation de Thomas d'Aquin engendrent des conceptions de l'homme et de sa fin appelées à s'opposer. Hertel, par son discours même, incarne ces tensions ; malgré sa facture thomiste, Pour un ordre personnaliste (1942), le deuxième essai de l'écrivain, embrasse un personnalisme qui incite les uns et les autres à se prononcer sur le bien-fondé d'une distinction entre l'individu et la personne.

\section{Abstract}

Like many interwar French Canadian thinkers, François Hertel was a Thomist. Indeed, the resurgence of St. Thomas Aquinas' philosopbical and religious thought 
left a profound mark on French Canadian Catholicism. However, in spite of appearances, Thomism was not monolithic in interwar French Canada. Differences in the interpretation of Aquinas' work generated opposing concepts about man and his ends. In his writing, Hertel embodied these tensions. Despite its Thomistic approach, Hertel's second book, Pour un ordre personnaliste (1942), embraced a personalism which gave rise to differing views regarding the validity of the distinction between the individual and the person.

Le Québec commémorait cet automne le vingtième anniversaire $^{1}$ de la mort de François Hertel, cet intellectuel catholique et nationaliste à l'avant-scène de la vie littéraire dans les années trente et quarante. De par son statut social, l'auditoire privilégié que constituaient ses classes, une activité intellectuelle intense, son originalité et son envergure, Hertel a exercé une influence dont on ne peut avoir idée aujourd'hui. "Les jeunes, surtout, ont peine à saisir ce qu'il a représenté pour nous. L'influence de Hertel est à peine imaginable pour ceux qui n'ont pas connu le monde, le Canada et le Québec d'avant $1960^{2}$ », témoigne Gérard Pelletier, l'un des fondateurs de Cité libre. Avant lui, Jean Éthier-Blais et Pierre de Grandpré estiment que « peu d'hommes auront joué, dans la vie intellectuelle du Canada français au vingtième siècle, un rôle aussi important que $\mathrm{Hertel}^{3}$ ». Dans ses mémoires, Pierre Elliott Trudeau dit de Hertel :

Hertel a été pour nous un initiateur exceptionnel dans plusieurs domaines. [...] Les auteurs moins fréquentés, français d'abord mais aussi anglais, américains, voire scandinaves, c'est Hertel qui me les a fait connaître. [...] C'est lui encore qui nous a traînés chez les artistes qui apprivoisaient alors Montréal à l'art contemporain. Pellan, Borduas et leurs disciples faisaient encore figure de marginaux mais Hertel fréquentait leurs œuvres de préférence à celles des peintres déjà consacrés. Il 
allait spontanément vers tout ce qui était nouveau ou à contre-courant des goûts du jour ${ }^{4}$.

Né le 31 mai 1905 à Rivière-Ouelle, François Hertel, de son vrai nom Rodolphe Dubé, passe son enfance dans le Bas Saint-Laurent, jusqu'à ce que la famille déménage à TroisRivières, en 1920. Au terme du cours classique, en 1925, Hertel décide de se faire prêtre et se présente au noviciat des Jésuites, à Montréal. Commence alors pour lui une formation de quinze ans, au cours de laquelle il enseigne aux collèges Jean-de-Brébeuf et Saint-Ignace. Hertel quitte la Compagnie de Jésus en février 1943 et devient prêtre diocésain à Montréal. Il enseigne au Collège Grasset, dirigé par les Sulpiciens, jusqu'en juin 1947. En 1949, Hertel s'installe à Paris de manière définitive. Par la suite il quitte les ordres, et reçoit son indult de laïcisation en novembre 1950. Au terme d'une existence bien remplie mais difficile en France, Hertel rentre au pays en juin 1985, et meurt quelques mois plus tard dans l'anonymat.

L'" ancien chef de file de l'intelligentsia québécoise ${ }^{5}$ » a innové dans plusieurs domaines, à titre d'essayiste, de romancier, de poète, de rédacteur de revues, d'éditeur, de chroniqueur philosophique ${ }^{6}$. Ses œuvres du début ont constitué, à divers titres, des premières, comme en ont rendu compte ses contemporains. Pour un ordre personnaliste, publié en 1942, est de l'inédit. D'après Guy Sylvestre, " cette somme personnaliste [...] est une des plus importantes contributions à la naissance d'une pensée canadienne ${ }^{7} »$. Marie-Joseph d'Anjou abonde dans le même sens, dans L'Action nationale: "on goûtera en liberté cette somme de philosophie et de théologie qu'est l'ouvrage de François Hertel, la première, dans tous les sens, dont s'honorent les lettres françaises au Canada ${ }^{8} »$. Un demisiècle plus tard, Jean-Claude Simard leur donne raison: «Ce texte $[. .$.$] constitue l'un des jalons certains de l'apparition d'une$ 
modernité philosophique au Québec ${ }^{9} »$. Tout autant que Leur inquietude (1936), le premier essai de Hertel, Pour un ordre personnaliste est un poste d'observation privilégié des années trente au Québec. L'essai philosophique restitue dans leur essence les discours des élites qui cherchent des remèdes à la crise morale dans laquelle les plonge un marasme économique sans précédent ${ }^{10}$. L'époque se caractérise par un catholicisme triomphant, auquel adhère sans réserve Hertel. Le jésuite fait preuve d'une fidélité sans faille à Rome et à l'Église, quoi que pensent ou disent ses détracteurs. Il remettra sa foi en cause plus tard, au début des années cinquante ${ }^{11}$. Le catholicisme vigoureux de l'entre-deux-guerres s'appuie sur un thomisme non moins robuste au Canada français, remis au goût du jour dans toute la chrétienté par le pape Léon XIII. La pensée québécoise gardera l'empreinte thomiste pendant toute la première moitié du $\mathrm{XX}^{\mathrm{e}}$ siècle - jusqu'au matin de la Révolution tranquille, avance le philosophe Jean-Paul Brodeur ${ }^{12}$. Hertel est résolument thomiste; le voudrait-il qu'il lui serait difficile de s'en défendre. Il venait à peine de terminer ses années de philosophie lorsque le provincial jésuite, Adélard Dugré, déclarait, en 1933, qu'« une des plus grandes ambitions des Jésuites de tous les temps» était «le triomphe des doctrines de saint Thomas d'Aquin dans le monde ${ }^{13}$ \%.

Une relecture de Pour un ordre personnaliste, est instructive à plus d'un égard : en plus d'éclairer sur l'état du catholicisme dans l'entre-deux-guerres, elle invite à considérer sous une perspective nouvelle le thomisme, tombé dans l'oubli au Québec, même s'il a constitué, de l'avis de Brodeur, " un très long épisode de l'histoire de notre activité philosophique», et qu'«aucun, au point de vue de son importance, ne puisse lui être comparé ${ }^{14} \%$. 
De plus, le thomisme, contrairement à ce que l'on pourrait croire, n'est pas uniforme au Québec. Brodeur met l'idée en avant :

Un malentendu persistant consiste à faire croire que le thomisme québécois constitue un champ de pensée homogène qui reçoit son unité de ce que tous ceux qui l'ont habité ont été fidèles à la pensée de saint Thomas. Or il n'est pas une seule des notions que l'on utilise pour caractériser le thomisme québécois qui ne doive être déconstruite $[\ldots]^{15}$.

Professé par l'élite intellectuelle canadienne-française, le thomisme est au même moment source de tensions, souterraines et difficiles à détecter, mais non moins réelles. La publication de Pour un ordre personnaliste, qui précède de quelques mois à peine celle de l'éminent philosophe de l'Université Laval, Charles de Koninck, De la primauté du bien commun contre les personnalistes. Le principe de l'ordre nowveau ${ }^{16}$, dévoile après coup cette réalité moins connue. Il faut, pour se faire une idée juste des fluctuations du thomisme canadien-français, s'arrêter à la restauration thomiste, engagée à la fin du XIX ${ }^{e}$ siècle. Il faut ensuite, dans l'hypothèse où les premiers essais de Hertel cristallisent les tensions au sein du thomisme d'ici, vérifier comment il se confirme que le jésuite lui-même est thomiste. Pour un ordre personnaliste, constatera-t-on à la même occasion, porte la marque de Jacques Maritain ; or ce dernier est l'un des principaux initiateurs du débat sur l'individu et la personne, qui allait causer des trépidations dans les cercles philosophiques canadiens-français et ouvrir une brèche dans la muraille thomiste. On découvrira de plus que Hertel est personnaliste, un fait de première importance : d'une part le personnalisme alimente de façon indirecte les discussions sur l'individu et la personne ; d'autre part, il est au nombre des raisons susceptibles d'avoir provoqué son départ des Jésuites, en 1943, et du pays, quelques années plus tard. 


\section{La restauration du thomisme}

L'Église catholique prête un intérêt accru à la question sociale, à la fin du XIX ${ }^{e}$ siècle; de plus, elle cherche à donner un nouvel élan à la recherche philosophique. Elle se tourne vers un grand penseur du Moyen Âge, Thomas d'Aquin. Au début de son pontificat, Léon XIII prône un retour au célèbre philosophe. Il lance l'appel suivant, dans l'encyclique Æterni Patris :

[...] Nous vous exhortons instamment [...] à rétablir et à propager autant que possible l'excellente doctrine de saint Thomas pour la défense et l'honneur de la foi catholique, pour le bien de la société, pour l'avancement de toutes les sciences. [...] Au reste que les professeurs choisis [...] s'attachent à faire pénétrer la doctrine de saint Thomas dans l'esprit de leurs élèves, qu'ils en mettent en évidence la solidité et l'excellence incomparable. Que les universités que vous avez fondées ou que vous fonderez l'expliquent, la défendent et l'emploient à la réfutation des erreurs contemporaines ${ }^{17}$.

L'encyclique d'août 1879 tenterait de contrebalancer l'influence «néfaste » de la philosophie moderne, qui «fournit des armes aux ennemis de l'Église ${ }^{18} »$. Rome se préoccupe de « la restauration chrétienne des discours scientifiques contaminés par ces grandes erreurs modernes que sont le rationalisme, le scientisme, le pragmatisme et le positivisme ${ }^{19}$ ", explique Yvan Cloutier, dans un article sur l'hégémonie philosophique dominicaine au Canada français.

Au Québec, la restauration thomiste est menée rondement, sous la houlette de prélats marquants tels Louis-Adolphe Pâquet et Jean-Marie-Rodrigue Villeneuve. Le premier, doyen de la Faculté de théologie de l'Université Laval pendant plus de trente ans (1904-1938), est considéré par plusieurs comme «le principal instaurateur du thomisme au Ca- 
nada $^{20}$ ». Le second, qui allait devenir archevêque de Québec puis cardinal ${ }^{21}$, déclarait à l'occasion de la fondation de l'Académie canadienne Saint-Thomas d'Aquin, en janvier $1930^{22}$, que « si l'Église est thomiste, et elle l'est indéniablement, c'est parce que Dieu le veut ainsi ${ }^{23}$ ». Les principales institutions d'enseignement supérieur emboîtent le pas avec empressement. Au Séminaire de Québec, on s'emploie à conformer l'enseignement de la philosophie à l'encyclique Æterni Patris un mois à peine après la promulgation de celle-ci, rappelait Maurice Roy à l'occasion des premières journées thomistes, à Ottawa $^{24}$. À la Faculté de théologie de l'Université Laval, on enseigne, dès septembre 1884, à partir de la Somme théologique de Thomas d'Aquin. À Montréal, enfin, les statuts de la Faculté de philosophie, approuvés par Rome en 1925, stipulent qu'« elle a pour but de donner l'enseignement supérieur de la philosophie selon la méthode, la doctrine et les principes du docteur Angélique ${ }^{25}$ ».

Sans doute est-il opportun d'introduire une parenthèse sur le thomisme comme tel. Le terme est dérivé de Thomas, Thomas d'Aquin en l'occurrence, un philosophe et théologien italien du XIII ${ }^{e}$ siècle $(122[5]-1274)^{26}$. Le dominicain a eu une influence considérable dans l'histoire de l'Église, et s'est imposé à travers les siècles par la force de sa philosophie, qui tient «à cette capacité de s'emparer du meilleur de la philosophie ancienne en la rapportant aux lois du christianisme, conciliant ainsi autant celle qu'on peut déduire des Pères de l'Église que celle de penseurs moins orthodoxes, comme Aristote ${ }^{27} »$, estime E.-Martin Meunier, auteur d'une thèse sur les transformations de l'éthique catholique au $\mathrm{XX}^{e}$ siècle. On doit en effet à saint Thomas d'avoir concilié la métaphysique du célèbre philosophe grec et la théologie chrétienne, au XIII ${ }^{\mathrm{e}}$ siècle. D'après Marie-Dominique Chenu, «la théologie de saint Thomas s'articule, se construit, sous la lu- 
mière d'une confiance active en la raison et d'une référence constante à la nature ${ }^{28}$ ». Sous l'impulsion de Thomas d'Aquin, la scolastique, qui cherchait « un accord entre la Révélation et la lumière de la raison ${ }^{29}$ ", explique Jacqueline Russ, connaît un réel essor. Le dominicain italien sépare nettement deux domaines, celui des vérités de raison et celui des vérités de foi, ajoute-t-elle ${ }^{30}$. L'immortalité de l'âme, la création du monde, appartiennent au deuxième domaine.

Dans les années où le thomisme était à son apogée au Québec, le philosophe Hermas Bastien en proposait la définition suivante :

Le thomisme, c'est la doctrine de la raison humaine, élaborée par le génie grec, repensée par le génie médiéval et catholique, dont l'impersonnalité universelle peut s'enrichir indéfiniment des découvertes de la science et de la spéculation philosophique ${ }^{31}$.

À la vérité, le thomisme, qu'on élabore depuis plus de 700 ans, ne se définit pas aisément, d'autant plus "qu'à peu près toutes les thèses essentielles de Thomas furent, au cours de l'histoire, soit contestées, soit ignorées par l'un ou l'autre "thomiste" ${ }^{\prime 2}$ ", explique l'auteur d'un essai sur l'histoire des thomismes, Géry Prouvost. Au XX siècle, les conflits d'interprétation se sont poursuivis ; qu'on songe, souligne-t-il, aux débats entre Maritain, Gilson, Garrigou-Lagrange ou Sertillanges ${ }^{33}$.

L'acquiescement de Hertel au thomisme est tout aussi clair que son adhésion au catholicisme ${ }^{34}$. Pour un ordre personnaliste est construit à la manière d'un cours de philosophie, en ce sens que chaque chapitre appartient à l'une ou l'autre des sections constitutives de tout manuel thomiste de base de l'époque ${ }^{35}$ : la métaphysique, la morale et l'esthétique. Le premier chapitre, par exemple, appartient à la métaphysique et aborde des points de psychologie et d'ontologie. 
Les chapitres sur le personnalisme social et le corporatisme, ceux sur la contemplation et le péché sont dans la sphère de la philosophie morale. Le chapitre sur le travail touche à l'esthétique, le travail d'après le sens que lui prêtaient les scolastiques, au Moyen Âge ${ }^{36}$. Le chapitre sur l'art, qui correspond lui-même à ce qu'on appelait naguère les beaux-arts, reprend des notions de psychologie (métaphysique) sur l'âme intellective ${ }^{37}$. Enfin, les deux chapitres de la dernière partie, sur la sainteté et l'incarnation, recoupent en plusieurs points la théodicée, une partie de la métaphysique. Hertel, il est vrai, néglige la logique, mais la charpente de son essai laisse apparaitre des bases solides dans le domaine. D'après ce dernier, nul mieux que saint Thomas ne saura guider la civilisation occidentale dans ses efforts pour "rendre la jungle humaine habitable ", conclut-il dans Pour un ordre personnaliste:

[...] et c'est à saint Thomas d'Aquin, le maitre de la lucidité consciente, le roi des philosophes réalistes qu'il ['homme d'aujourd'hui] va redemander, avec les commentaires d'Aristote éclairés des lumières du dogme, des raisons et une vision plus large dans la reconstruction d'une civilisation que trop de snobismes ont dévoyée dès son origine $e^{38}$.

Tout thomiste qu'il soit par ailleurs, Hertel manie les concepts avec suffisamment de souplesse pour se sentir autorisé à épouser les opinions de certains personnalistes français. Il adopte d'eux une idée qui leur est chère : la personne humaine comme valeur suprême.

\section{Hertel, thomiste... et personnaliste}

À l'instar des personnalistes, Hertel croit qu'un véritable ordre de vie place au premier rang les personnes et que tout doit converger vers elles. «La personne [...] est le centre du monde ${ }^{39}$ ", affirme-t-il. Non seulement rallie-t-il un mou- 
vement propre aux années trente, Hertel suit un courant de pensée qui a traversé tout le siècle. "L'affirmation du caractère sacré, transcendant et inviolable de la personne représente assurément l'une des grandes mutations de l'éthique catholique au $\mathrm{XX}^{\mathrm{e}}$ siècle $^{40}$ ", note Meunier. En Europe, le mouvement personnaliste est "d'abord le "cri de ralliement" d'une génération de jeunes intellectuels en face des défis d'une époque qui devait s'avérer un tournant dans l'histoire de la France, de l'Europe, et même de l'Occident ${ }^{41}$ ", note Jean-Louis Loubet Del Bayle dans une étude sur les non-conformistes de l'entre-deux-guerres. Il faut chercher une voie "par-delà le fascisme, le communisme et le monde bourgeois décadent ${ }^{42}$ ", affirme dans un manifeste l'un des chefs de file du personnalisme, Emmanuel Mounier.

Comme c'était aussi le cas pour le thomisme, il est difficile de définir le personnalisme. Dans une perspective très large, on pourrait avancer que tout philosophe chrétien est personnaliste, puisque le personnalisme est « historiquement d'origine chrétienne ${ }^{43}$ ", comme l'affirme Jean-Marie Grevillot. Mounier en donnait la définition suivante, en 1936: "Nous appelons personnaliste toute doctrine, toute civilisation affirmant le primat de la personne humaine sur les nécessités matérielles et sur les appareils collectifs qui soutiennent son développement ${ }^{44}$. » Il prévient toutefois que le "personnalisme n'annonce pas la constitution d'une école, l'ouverture d'une chapelle, l'invention d'un système $\operatorname{clos}^{45}$ ». Maritain abonde dans ce sens :

Rien ne serait plus faux que de parler du « personnalisme» comme d'une école ou d'une doctrine. [...] Il n'y a pas une doctrine personnaliste, mais des aspirations personnalistes et une bonne douzaine de doctrines personnalistes, qui n'ont parfois en commun que le mot de personne [...]. Il y a des personnalismes à tendance proudhonienne, des personnalismes qui penchent 
vers la dictature et des personnalismes qui penchent vers l'anarchie ${ }^{46}$.

Sa conception du thomisme comme d'un "système en mar$\mathrm{che}^{47}$ ", de même que son admiration pour Maritain et Mounier conduisent naturellement Hertel au personnalisme. Il s'appliquera à accorder le personnalisme avec la démarche de Thomas d'Aquin, qui lui semble encore «le meilleur docteur du personnalisme ${ }^{48}$ ", explique-t-il dans une lettre inédite à Sylvestre : «J'ai voulu montrer que [...] le personnalisme pouvait être considéré comme une pure et simple doctrine thomiste, comme une thèse thomiste dérivée, si vous préfére $z^{49}$. " Il prétend qu'à sa connaissance, un tel rapprochement n'avait pas été fait. "Dans Humanisme intégral, souligne-t-il, Maritain n'a pas pris la peine de montrer que la philosophie personnaliste est parfaitement thomiste ${ }^{50}$. "Il est vrai que Maritain n'est pas aussi systématique dans son célèbre essai. Il n'en demeure pas moins qu'il y affirmait que « [...] la théologie de saint Thomas dominerait une nouvelle chrétienté ${ }^{51} »$. Bien avant Hertel, Maritain déclarait être intéressé par un personnalisme "fondé sur la doctrine de saint Thomas $\mathrm{d}^{\prime}$ Aquin ${ }^{52}$ ». Meunier estime de plus que "par sa critique du monde moderne, Maritain fut l'un des premiers catholiques français du siècle à proposer les prémisses philosophiques d'un personnalisme fortement inspiré de Thomas d'Aquin ${ }^{53}$ ». Mais l'association $\mathrm{du}$ thomisme et du personnalisme ne recueille pas l'adhésion de tous, car elle soulève l'épineuse question des droits individuels et collectifs : le bien de la personne, comme on disait alors, peut-il avoir préséance sur le bien commun, et si oui, dans quelles conditions ? Nous y reviendrons à la section suivante.

Hertel fut sans aucun doute plus influencé par Maritain, qu'il appelle " notre maître ", dans sa lettre à Sylvestre ${ }^{54}$, que par Mounier. Né en 1882, converti au catholicisme en 
1906, Maritain jouit d'un prestige incontestable au Canada français, rappelle Cloutier :

La diffusion des conférences de $1934^{55}$ [de Maritain] élargit à un grand public une influence déjà considérable sur le clergé et dans les milieux de jeunesse depuis les années 1920. La réception de Maritain dans la Revue dominicaine et L'Action nationale atteste une solide réputation de philosophe. Maritain est bien reçu par les milieux nationalistes et conservateurs et par les groupes plus libéraux ${ }^{56}$.

Non seulement «le capital symbolique de Maritain atteint-il son apogée dans les années 1920 et $1930^{57}$ », il « se maintient jusque dans les années $1940^{58}$ ", assure Cloutier. Certaines autorités expriment publiquement leur admiration pour Maritain, dont Lionel Groulx : "On le voit intervenir dans les moments plus graves de la vie contemporaine pour rectifier les idées, ériger au-dessus de la mêlée des faits, l'astre lumineux des principes ${ }^{59}$ ", écrit-il dans une recension de Primauté du spirituel. Dans Itinéraires philosophiques, Bastien consacre tout un chapitre à Maritain, saluant chez lui «les qualités d'apôtre désireux que la vérité dans les âmes pousse les volontés à l'action ${ }^{60} \%$.

L'influence de Maritain sur l'œuvre de Hertel est indéniable ${ }^{61}$, et nulle part plus manifeste que dans Pour un ordre personnaliste ${ }^{62}$. Par exemple, le chapitre portant sur le travail renvoie le lecteur à Art et scolastique, paru en 1920. Le chapitre sur la contemplation est proche de Primauté du spirituel, un essai publié par Maritain en $1927^{63}$. La première partie de Pour un ordre personnaliste emprunte à Humanisme intégral, imprimé et distribué au Québec en 1939 par les Éditions de l'Arbre. De plus, Hertel reproduit fidèlement dans son essai, à la page 90, la définition que donne Maritain d'une civilisation personnaliste. Deux idées directrices chez Maritain, à savoir 
qu'il y a au sein de cette civilisation personnaliste une œuvre commune à réaliser et une œuvre personnelle de salut, se retrouvent dans l'essai de Hertel ${ }^{64}$.

Par ailleurs, quiconque se rallie au personnalisme doit participer à l'échange de vues sur l'individu et la personne et exposer tôt ou tard sa position sur la question. Hertel se plie lui aussi à cet impératif. Une analyse de son point de vue s'impose non seulement parce qu'elle le situera face à ses pairs, mais aussi parce que sa position aura, est-il permis de supposer, des incidences sur son avenir au sein de la Compagnie de Jésus.

\section{L'individu et la personne, au cœur des débats dans les années 1930}

Dans l'entre-deux-guerres, on discute avec passion de l'individu, de la personne et de la société, des droits et devoirs respectifs de chaque partie; «le rapport individu et société est particulièrement préoccupant étant donné la montée et l'extension planétaire des divers fascismes et totalitarismes ${ }^{65}$, explique Simard. Le débat sur l'individu et la personne se transporte dans les cercles philosophiques catholiques : « au cours de ces années 30 , c'est une opposition qui revient fréquemment sous la plume de penseurs catholiques dont la doctrine se situe dans le prolongement direct de celui de saint Thomas $^{66}$ ", note Roger Benjamin dans une étude sur le personnalisme chrétien. Il faut alors revenir à Maritain, dans une bonne mesure à l'origine du débat ${ }^{67}$. Le philosophe fait une distinction entre l'individu et la personne dans Trois réformateurs :

Selon l'enseignement de saint Thomas, [...] l'homme tout entier comme individu [...] est ordonné au bien de la cité comme la partie est ordonnée au bien du tout, au bien commun [...]. Mais s'il s'agit de la personne comme 
telle, le rapport est inverse, et c'est la cité humaine qui est ordonnée aux intérêts éternels de la personne et à son bien propre, lequel est, en fin de compte, [...] Dieu lui-même $;[\ldots]^{68}$.

Simard résume bien la position de Maritain : "Selon lui, le bien et les devoirs de l'individu sont subordonnés à ceux de la société, mais elle-même doit préséance à la personne ${ }^{69}$. " La différenciation introduite par Maritain donnera naissance à la formule "l'individu pour la société, la société pour la personne », rappelle Benjamin ${ }^{70}$.

Or le fondement thomiste d'une telle distinction a été "parfois contesté ${ }^{71}$ ", note Loubet Del Bayle. Pour les défenseurs du bien commun, le véritable bien de la personne est indissolublement lié à la réalisation du bien commun - et c'est dans ce sens que ce dernier serait supérieur au bien privé. Faire du bien privé le bien suprême néglige, dans leur esprit, ce lien, et évoque trop une conception libérale et individualiste de la personne et de la société. De là tout le débat. Pourtant, Maritain se défend de verser dans l'individualisme, ce que craignent en effet certains thomistes :

Ainsi en chacun de nous l'individu est pour la cité, et doit au besoin se sacrifier pour elle, comme il arrive dans une juste guerre. Mais la personne est pour Dieu ; et la cité est pour la personne [...]. Disons que la Cité chrétienne est aussi foncièrement anti-individualiste que foncièrement personnaliste ${ }^{72}$.

Hertel, faut-il se demander, épouse-t-il le point de vue de Maritain ? À l'instar du philosophe français, il se défend d'être individualiste : "Qu'on nous comprenne bien toutefois : notre souci de revenir à l'homme, corps et âme, à l'homme personne, est à cent lieues d'une conception matérialiste ou individualiste ${ }^{73}$ ", se récrie-t-il. Hertel partage le point de vue de Maritain, à cette nuance près qu'il paraît moins catégorique : 
s'il établit une différence entre l'homme "personne » et l'homme "individu», Hertel ne manque jamais de rappeler que les deux notions sont inséparables:

Il y a une œuvre commune à réaliser par toutes les personnes considérées comme individus d'une même espèce ; il y a aussi œuvre personnelle de salut qui n'intéresse qu'une personne à la fois [...]. Ces deux points de vue de la personne ne sont point séparables. [...] nous devrons les garder constamment en mémoire ${ }^{74}$.

De plus, Hertel précise que «la parenté des corps [la société, pour Hertel] devra souvent céder le pas aux intérêts supérieurs des âmes, aux intérêts des personnes ${ }^{75}$ " - «souvent», pas toujours, doit-on comprendre. Mais pour Maritain, la préséance de la personne sur l'individu semble automatique : « s'il s'agit de la personne comme telle, [...] c'est la cité humaine qui est ordonnée aux intérêts éternels de la personne et à son bien propre, lequel est, en fin de compte, [...] Dieu lui-même ${ }^{76}$ ", soutient-il.

S'il fallait, en raison de l'importance de Maritain et du retentissement de ses écrits, lui comparer Hertel, il convient de se demander comment se présentait le débat sur l'individu, la personne et le bien commun au Québec. Hertel, proche de Maritain, défendait-il une position à laquelle souscrivaient les élites canadiennes-françaises ? À l'époque, il est l'un des rares défenseurs du mouvement personnaliste au Canada français. Peu d'intellectuels de sa génération ont écrit de manière aussi substantielle sur le sujet. Les revues $L a$ Relêve, La Nouvelle Relève et plus tard Cité libre, furent, il est vrai, des foyers du personnalisme, comme le souligne Christian Roy ; ce dernier fait le parallèle suivant entre le personnalisme canadien-français et celui de France :

Comme les personnalistes d'outre-mer, ils [les personnalistes québécois] voulaient une révolution qui 
fût d'abord spirituelle ; [...]. Néanmoins, à la différence des personnalistes français, ils se bornaient à scruter la racine métaphysique [...], sans guère se poser la question des moyens d'action concrets et des changements institutionnels qui hantait leurs maitres ${ }^{77}$.

Hertel lui-même, faut-il noter, n'appelle pas à l'action politique ; il écrit, dans l'avant-propos de Pour un ordre personnaliste:

Les jeunes d'âge et ceux dont la destinée est de ne pas vieillir n'ont pas démissionné. [...] La plupart ont renoncé pratiquement à la conquête politique. [...] D'autres commencent d'affirmer que le véritable redressement devra se faire dans le domaine culturel et spirituel. Quand ce point sera mieux compris, ce sera la victoire définitive ${ }^{78}$.

Les idées de Hertel s'accordaient-elles avec celles des autorités de l'époque, dont il ne s'est jamais véritablement démarqué ? La réponse la plus sûre, peut-être la seule à laquelle autorisent les limites du présent article, est que le point de vue de Hertel ne faisait pas l'unanimité.

Le personnalisme suscitait des réticences chez certains philosophes canadiens-français, et non des moindres, à cause notamment de la primauté du bien commun, un principe avéré sur lequel aucune concession n'était permise. «Les voix n'ont pas été unanimes dans l'école scolastique [...] pour admettre la possibilité d'un personnalisme thomiste ${ }^{79}$ ", affirme l'auteur d'une étude sur les fondements thomistes du personnalisme de Maritain, Jacques Croteau. Hertel est du reste le premier à le reconnaître : "le personnalisme était considéré et l'est encore comme une doctrine peu conciliable avec la philosophie thomiste ${ }^{80} \ldots$ "., écrit-il à Sylvestre.

Hertel, par certaines affirmations, se rapproche pourtant des scolastiques stricts, au nombre desquels on pourrait ranger Henri Grenier, professeur au Séminaire de Québec et 
l'auteur de Cours de philosophie ${ }^{81}$. Il y a lieu en effet de douter que ce dernier eût enseigné au Séminaire, s'il n'avait reçu la bénédiction de l'expert en philosophie scolastique qu'était alors $\mathrm{M}^{\mathrm{gr}}$ Pâquet. Hertel, lorsqu'il écrit que «nulle société n'a le droit de toucher aux droits inaliénables de la personne ${ }^{82}$ », va dans le même sens que Grenier : il est admis sans discussion, chez les philosophes scolastiques, affirme en effet ce dernier, que « chaque personne humaine et chaque famille conservent, dans la société civile, les droits que la nature leur a donnés ${ }^{83}$ ”. Hertel ne contredit pas non plus Grenier, lorsqu'il soutient que la personne humaine transcende la société "grâce à sa personnalité spirituelle ${ }^{84} »$; Grenier prétend lui aussi que «la fin surnaturelle de l'homme ou de la personne humaine est un bien supérieur à la fin de la société civile ${ }^{85}$. » Mais en dernière analyse, Grenier n'en associe pas moins le personnalisme à l'individualisme: "Nous pouvons [...] dire que le personnalisme est une forme d'individualisme. En effet, il subordonne, en définitive, le bien commun, le bien de la multitude, au bien des personnes qui constituent la multitude, c'est-à-dire au bien de l'individu ${ }^{86} »$, conclut-il. La fin personnelle de l'homme est au contraire " ordonnée au bien commun, comme la partie l'est au tout ${ }^{87}$, estime Grenier.

D'autres opposants au personnalisme prennent la plume : parmi ceux-ci l'influent dominicain Louis Lachance, auteur de L'bumanisme politique de saint Thomas: individu et état (1939) :

[...] nous nous refusons à prendre à notre compte la théorie personnaliste mise en cours dans ces derniers temps et censée dévoiler l'orient de la coordination thomiste. Il nous fut impossible d'en trouver quelque fondement sérieux dans les écrits de $\mathrm{S}$. Thomas, et nous craignons que, trop logiquement articulée, elle doive aboutir à des erreurs périlleuses ${ }^{88}$. 
Mais l'attaque la plus acharnée contre le personnalisme est déclenchée par Charles de Koninck, dans son livre De la primauté du bien commun contre les personnalistes. Le principe de l'ordre nouvean ${ }^{89}$. Dans la première de deux dissertations (la seconde portant sur le marxisme), le professeur, très écouté par l'épiscopat québécois, défend «la primauté du bien commun contre ceux qui voudraient, à son détriment, centrer la société sur la personne humaine ${ }^{90} "$, résume Frédéric Saintonge dans Relations. De Koninck craint que «l'exaltation de la personne ne tourne toujours à l'exaltation du bien privé ", et il "n'admet aucune subordination du bien commun au bien privé de la personne ${ }^{91}$ ", explique Croteau. Dans la préface à De la primauté du bien commun contre les personnalistes, une autre autorité de Québec, le cardinal Villeneuve, cautionne de Koninck:

Présentement, c'est le personnalisme qui est à la mode. Des esprits très sincères le préconisent. On exalte la dignité de la personne humaine, on veut le respect de la personne, on écrit pour un ordre personnaliste, on travaille à créer une civilisation qui serait pour l'homme... Tout cela est très bien, mais trop court, car la personne, l'homme, n'est pas sa fin à elle-même ni la fin du tout ${ }^{22}$.

D'autres intellectuels partagent l'avis de Son Éminence. De Koninck «fait voir avec une évidence à crever les yeux que l'une des conséquences les plus immédiates du personnalisme est précisément ce totalitarisme barbare et inhumain que les personnalistes prétendent combattre au nom de la liberté et de la dignité de la personne ${ }^{93}$ "), écrit Eugène Babin. D'après lui, De la primauté du bien commun contre les personnalistes déplaira sûrement aux thomistes «à la main tendue, sans cesse agités du désir de composer avec la pensée moderne ${ }^{94} \%$.

Il est difficile de déterminer avec certitude si de Koninck vise directement Hertel dans son livre. Cloutier répond par 
l'affirmative, en ce qui a trait du moins à la préface : «La préface de Villeneuve pointe aussi en direction du jésuite montréalais François Hertel dont les Éditions de l'Arbre avaient publié à l'automne 1942 Pour un ordre personnaliste ${ }^{95}$ ", soutient-il. Avant Cloutier, Yves Simon reconnaissait également que De la primauté du bien commun contre les personnalistes pouvait inciter le lecteur à englober Hertel dans les personnalistes fustigés par de Koninck; en définitive, concluait-il cependant, on tendrait plutôt à croire que Maritain était la personne ciblée :

Demandons-nous quels noms viennent spontanément à l'esprit du lecteur typique [...] lorsqu'il est fait mention des personnalistes [dans de Koninck]. [...] $[\mathrm{Q}]$ uelques auteurs canadiens-français, dont le plus connu est François Hertel, l'auteur de Pour un ordre personnaliste [...] ; en dernière analyse, en raison de l'importance de la personne dans les travaux de Maritain, il y a lieu de croire que l'expression " les personnalistes " correspond, dans l'esprit du lecteur, à Maritain ${ }^{96}$.

De Koninck aurait admis lui-même avoir eu Maritain comme cible principale, dans une lettre à un confrère, en 1946, affirme Cloutier ${ }^{97}$.

Au Canada français comme ailleurs, constate-t-on, le thomisme n'est pas un tout lisse et homogène. Les parutions consécutives des essais de Hertel et de de Koninck en sont la meilleure illustration. Le livre de ce dernier, note Babin dans Culture, "montre que la division chez les thomistes est aujourd'hui beaucoup plus profonde et plus universelle qu'on ne veut l'admettre en quelques milieux ${ }^{98} »$. Pour paraphraser Maritain lorsqu'il parle du personnalisme, il n'y a pas un thomisme canadien-français, mais des thomismes canadiens-français. 
D'après Hertel, le thomisme «n'est pas un système fixé [...] et n'aura pas (même de nos jours) la même couleur en France, en Italie, en Allemagne ${ }^{99} »$. Une telle conception lui a permis d'obliquer vers le personnalisme. Peut-on en déduire pour autant que les autres philosophes canadiens-français, par comparaison, pratiquaient un thomisme figé, qui aurait donné lieu à des " excès scolastiques » et se serait transformé en une "orthodoxie contraignante», comme cela aurait été le cas en Europe, de l'avis de certains historiens du christianisme ${ }^{100}$ ? Hertel, faut-il le préciser, n'est pas le seul à croire que saint Thomas doit être "un phare et non une borne", selon l'expression de Bastien : «Le thomisme, écrit ce dernier, ne doit donc pas rétrécir l'horizon mais l'élargir. Rien ne ressemble moins à son influence logique, que le développement d'une mentalité étroite, inhospitalière, hostile à toutes les découvertes de la pensée moderne ${ }^{101}$.»

$\mathrm{M}^{\mathrm{gt}}$ Pâquet, par contre, "sacro-saint, tabou, intoucha$b^{102}$ " dans les années vingt, serait d'obédience ultramontaine, de l'avis d'Yvan Lamonde :

[...] la doctrine de Pâquet possédait toutes les garanties de l'orthodoxie. Appuyés sans cesse sur un thomisme romain, ses principes étaient sanctionnés par les encycliques et les documents pontificaux et leur interprétation demeurait celle des canonistes orthodoxes [...]. [...] Orthodoxe, le corps de la doctrine de Pâquet fut importé de l'étranger, sur l'axe ultramontain QuébecRome. En ce sens, Pâquet perpétuait sous de nouvelles formes la tradition ultramontaine ${ }^{103}$.

En fouillant dans leurs souvenirs, des anciens des collèges classiques se rappellent que l'analyse d'une multiplicité de points de vue n'était pas expressément encouragée, dans les années trente. Jean Éthier-Blais, collégien à cette époque, se remémore l'ambiance des classes de philosophie : 
Nous devions nous contenter de l'insipidité de Grenier [cf. ci-dessus]. La méthode d'approche ressemblait plus à un conditionnement militaire qu'à un approfondissement métaphysique. Nous apprenions par cœur syllogismes et explications, qui étaient à la fois tarabiscotés et simplistes. [...] Le thomisme était du côté des anges et des Canadiens français, gardiens irréprochables de la doctrine ${ }^{104}$.

Dans Nous ferons l'avenir, Hertel tient des propos approchants :

Nous nous croyons thomistes en philosophie, parce que nous étudions dans des manuels thomistes. Pourtant, le thomisme rajeuni, enrichi des apports de la philosophie moderne, le thomisme à la Maritain, à la Sertillanges, à la Claudel est trop dense pour nos esprits courts et morceleurs ${ }^{105}$.

La pensée thomiste prend plusieurs teintes, au Canada français, selon qu'on est de la génération de $\mathrm{M}^{\text {gr }}$ Pâquet ou de Hermas Bastien, qu'on a étudié à Rome ou non, qu'on est jésuite ou dominicain, qu'on collabore à Culture ou à Relations, qu'on habite Montréal ou Québec. On attend toujours une histoire du thomisme québécois, d'Æterni Patris à la Révolution tranquille.

\section{Hertel marginalisé malgré lui ?}

Hertel ne déroge pas à la loi non-écrite du milieu philosophique canadien-français et professe un thomisme fidèle, pour l'essentiel, à celui que l'on trouverait dans tout manuel ayant obtenu l'imprimatur. Il est également de son époque en participant au grand débat sur la personne et l'individu. Mais il y a lieu de se demander si sa position personnaliste, plus précisément sa conception du thomisme qui l'ouvre aux idées de Maritain sur la personne, ne le mettra pas éventuellement en marge, malgré lui. La question se pose, à la lumière des 
admonestations de son supérieur, le père Papillon, quelques mois avant la parution de Pour un ordre personnaliste; on reproche à Hertel de créer de petits cénacles, de trop écrire et surtout, d'interpréter un peu trop librement saint Thomas d'Aquin :

C'est une influence dangereuse [...] que celle d'un jésuite qui, au temps où nombre d'esprits supérieurs en quête de la vérité, beaucoup de protestants aussi, reviennent à l'étude de saint Thomas et de la scolastique, donne aux jeunes le dégoût de cette même scolastique $^{106}$.

Hertel, pour mémoire, quittera la Compagnie de Jésus six mois après cette lettre, en février 1943. Son exclusion, par la suite, subtile ou brutale selon les points de vue, personne ne s'aviserait de la mettre en doute. Son départ des Jésuites n'a pas été sans conséquences. Quelques mois à peine après celui-ci, ses textes ne paraissent plus dans L'Action nationale, une revue à laquelle il collaborait depuis 1935. "À l'époque, [...] sa sécularisation fit scandale ${ }^{107}$ ", affirme un ami, Paul Toupin. Elle a sûrement influencé le jugement du milieu littéraire, constate-t-on à la lecture de l'extrait suivant de Littérature canadienne-française, paru en 1954, où Hertel est décrit notamment comme un "refoulé qui tremble du dedans »:

[...] depuis 1949, c'est le drame sombre et triste qui le fait " jeter la soutane aux orties» (Berthelot Brunet). C'est le grand naufrage ; les livres, qu'il écrit depuis, alignent des témoignages inquiétants sur le malheur de ce naufragé, lucide, " noyé dans ses tourments ", dont le rire amer est plus poignant que plaintes et sanglots ${ }^{108}$.

Par ailleurs, le projet d'université libre ${ }^{109}$ de Hertel tombe vite à l'eau, "le pouvoir ecclésiastique n'étant certes pas disposé à le bénir ${ }^{110}$ ", explique Jean Tétreau. Selon ce dernier, Hertel ne réussissait plus à trouver de salle libre, en 1947, pour pro- 
noncer des conférences. Plus tard, Hertel lui-même soutiendra publiquement qu'à la même époque, on défendait aux libraires d'exposer ses livres dans la vitrine. «Il y avait des pressions. On me cachait. Il n'y a qu'Henri Tranquille qui ait pris le risque de me mettre en librairie ${ }^{111}$ ", déclare-t-il en entrevue. Les unes après les autres, les portes se ferment à l'ancien jésuite. Hertel décide de partir. Pour la France. «Il aura, en choisissant de vivre à Paris, "sa reine ", déserté son propre enterrement dans le Québec conformiste des années quarante $^{112} "$, conclut Yolande Grisé.

On ne peut affirmer pour autant que l'adhésion de Hertel au personnalisme soit seule en cause. La parution de Pour un ordre personnaliste ne pouvait sûrement pas laisser croire en effet qu'on ferait grief à Hertel de ses idées : au nombre des dix-huit critiques compulsées à des fins de vérification, une seule était nettement défavorable à l'essai de Hertel, soit celle d'un étudiant, Roberval Paradis ${ }^{113}$. Si l'on persiste à croire que ses opinions personnalistes ont discrédité Hertel, sans doute faudrait-il se reporter au débat sur l'individu et la personne, qui l'opposait - indirectement, peut-être - à des hommes de poids tels le cardinal Villeneuve, de Koninck et le dominicain Louis Lachance. Les aspects à considérer pour déterminer les causes de la mise au ban de Hertel — dans l'hypothèse toujours où celle-ci fut réelle - sont trop nombreux pour être analysés de façon concluante dans ces pages. Nous devons nous satisfaire d'hypothèses, qui n'en présentent pas moins un intérêt réel.

En s'engageant sur la voie personnaliste, Hertel, a-t-on remarqué, s'est rapproché de Maritain. On ne saurait dire si son admiration pour le philosophe français, dont il a repris un certain nombre d'idées, a joué contre lui. Elle a peut-être rangé Hertel parmi les philosophes suspects de transgresser l'orthodoxie. L'hypothèse a quelque crédit. Jean Éthier-Blais rap- 
pelle que «dans le domaine des idées, celles de Maritain paraissaient dangereuses ${ }^{114}$ ", dans les années trente. En dépit du grand prestige dont il jouissait chez les philosophes, Maritain n'obtenait pas non plus un assentiment unanime sur le plan de la politique. Sa position, lors de la guerre civile d'Espagne $^{115}$, était de nature à irriter certaines autorités ecclésiastiques, au Québec. Pour preuve, on lit dans L'Action catbolique, en mars 1941, que «l'opinion du grand philosophe sur la guerre civile espagnole [...] fut même combattue chez les Canadiens de langue française $\mathrm{e}^{116} \%$. Hertel, quant à lui, se distinguait de Maritain, souhaitant, dans Leur inquiétude, la création d'un parti catholique véritable, "comme celui de Gil-Robles en Espagne, qui a contenu pour un temps les forces de gauche », et qui finirait, espérait-il, par avoir le dessus ${ }^{117}$.

De plus, Maritain, sans mettre en doute le patriotisme « incontestable » de Pétain, n'a pas moins exprimé vigoureusement son opposition aux décisions du régime de Vichy: "Ce qui est stupéfiant, c'est que le gouvernement Pétain ait déclaré honorables, et donc tenu pour acceptables en euxmêmes, les termes de l'armistice qu'il était réduit à signer ${ }^{118}$ ", écrivait-il en 1940. De tels propos, reproduits en exclusivité dès janvier 1941 dans la Revue dominicaine, pouvaient indisposer certains membres de l'élite canadienne-française, fidèles à Pétain. Un mois à peine après la parution de ces extraits $\mathrm{d}$ ' $A$ travers le désastre, le cardinal Villeneuve tenait les propos suivants sur le maréchal, lors d'une messe solennelle Tempore Belli à la basilique Notre-Dame (Montréal) : "Nous vénérons l'auguste et noble vieillard qui tient en ce moment d'une main prudente, mais sans vaciller, les destinées de la nation qui fut celle de nos pères et pour laquelle nos cœurs battent toujours ${ }^{119}$.» Hertel, pour sa part, omet de mentionner Pétain dans ses essais. On ne retrace aucun texte où il se soit prononcé sur le régime de Vichy. Tout au plus espère-t-il qu'au lende- 
main de la guerre, en France, « le malheur aura apaisé les vieux griefs, et que le besoin de reconstruire entraînera l'union des volontés ${ }^{120} »$.

Enfin, Maritain est pris à partie à deux reprises dans $L e$ Devoir, en mai 1943 : une première fois le 3 mai, pour s'être porté à la défense du mathématicien français Jacques Hadamard, venu prononcer des conférences au Canada et accusé d'être "antireligieux et maçonnique ${ }^{121}$ ». Le 15 mai, Le Devoir publie en première page une critique très dure à l'endroit de Maritain, sous la plume du bénédictin français Albert Jamet ${ }^{122}$. Ce dernier reproche à l'auteur d'Humanisme intégral de détester Franco « autant qu'il déteste Pétain ${ }^{123}$ ». Les prises de position de Maritain, on le constate, pouvaient provoquer des réticences à l'endroit de ses thèses philosophiques, quelles qu'elles soient. Quarante ans plus tard, Jean Le Moyne fait le commentaire suivant, révélateur de la réputation dont semblait jouir Maritain à un certain moment au Québec :

La guerre ne rendra pas plus éclairée la province de Québec qui se sentait assez bien nantie (Gérard Filion la croyait surtout «bête ») pour se passer d'un Jacques Maritain, d'un Étienne Gilson [...]. Le scandale demeure et il importe d'en écrire l'histoire $[\ldots]^{124}$.

Maritain, s'il était susceptible d'exacerber certaines susceptibilités, comptait, faut-il rappeler, beaucoup d'admirateurs au Canada français. Il est difficile dès lors de mesurer l'impact des prises de position du philosophe français sur le plan politique. Peut-être ne réussit-on pas à départager aisément admirateurs et détracteurs pour la simple raison que plusieurs personnes étaient les deux à la fois : on aimait Maritain pour certaines raisons, on le critiquait pour d'autres. En conséquence, on ne saurait affirmer en toute certitude que son admiration pour Maritain a nui à Hertel, d'autant plus que ce dernier n'a endossé publiquement aucune des opinions politi- 
ques du philosophe français sur l'Espagne, Hadamard ou Pétain. De plus, il faudrait examiner de près les prises de position respectives de l'Église québécoise et des Canadiens français face à la France au long de la Deuxième guerre mondiale, afin de déterminer si elles ont pu avoir pour effet de stigmatiser les écrits de Hertel. Dans quelle mesure les uns et les autres ont-ils été pétainistes, ou gaullistes ? Par exemple, le même cardinal Villeneuve qui accordait en chaire son appui à Pétain, en février 1941, aurait été, de l'avis d'Éric Amyot, l'un des plus solides alliés de Charles de Gaulle au Québec ${ }^{125}$.

Hertel affiche un point de vue sur la personne et l'individu qui se distancie quelque peu de l'orthodoxie, pourraiton croire, mais on ne peut prouver hors de tout doute que ses idées et l'accueil qu'elles reçurent le conduiront à quitter les Jésuites, puis le Québec. Hertel est un disciple avoué de Maritain, mais là encore, rien ne permet de conclure de façon définitive que cela lui causera préjudice. Dans les deux cas subsistent des questions, cependant, auxquelles il reste à trouver réponse.

\section{$* * *$}

Hertel est thomiste dans l'entre-deux-guerres, comme l'époque et comme ses pairs. Son deuxième essai, Pour un ordre personnaliste, est probant à cet égard. Il se compare à tout manuel de philosophie de base, le chapitre sur la logique en moins. L'essai témoigne également de l'adhésion de Hertel au personnalisme - à un certain personnalisme, devrions-nous préciser, canadien-français, catholique, envisagé dans une perspective néo-thomiste, fondé sur la primauté du spirituel, marqué par Maritain.

La relecture de Pour un ordre personnaliste transporte le lecteur en des lieux moins connus de l'histoire de la philosophie canadienne-française, dans des chapelles thomistes dres- 
sées parfois l'une contre l'autre. Les uns estiment que le bien commun doit primer le bien privé de la personne, en toutes circonstances, et déclarent la guerre à l'individualisme. Les autres ne récusent pas cette idée, s'il est question de l'individu. Mais s'il s'agit de la personne, Maritain, et, dans une moindre mesure, Hertel, croient que la société doit plutôt être ordonnée aux intérêts de celle-ci. Hertel estime, à l'instar de Maritain, que la société doit placer la personne au premier rang. Mais il ne va pas aussi loin que son «maittre ", se contentant d'affirmer que la société devra souvent — pas toujours - céder le pas aux personnes. S'il adopte un point de vue personnaliste, Hertel ne s'éloigne pas pour autant, contrairement à ce que l'on pourrait croire, de l'orthodoxie, sachant faire preuve de prudence sur le plan philosophique. En clair, Pour un ordre personnaliste est un manuel de philosophie thomiste en ce sens qu'il est conçu de la même manière que celui-ci ; mais les plus orthodoxes pouvaient croire, à la sortie du livre, que le deuxième essai de Hertel s'éloignait du thomisme du fait de ses sources d'inspiration.

Si Hertel avait été marginalisé en raison de ses vues personnalistes, ce l'aurait été bien malgré lui, car il croyait avoir réussi à démontrer que le personnalisme pouvait être considéré comme " une pure et simple doctrine thomiste ». Il faut peut-être chercher du côté de Maritain une explication à l'exclusion de Hertel, dans les années qui suivirent la parution de Pour un ordre personnaliste. En dépit de son prestige auprès des intellectuels canadiens-français, Maritain affichait des opinions politiques susceptibles de déplaire dans certains milieux. La méfiance qu'il pouvait susciter s'est peut-être exercée sur Hertel, l'un de ses disciples déclarés. L'hypothèse mérite d'être examinée de près

Pour quelque raison que ce soit, Hertel a fait les frais, dans une certaine mesure, des tensions au sein de la famille 
thomiste, exacerbées en ces années de guerre. Il a peut-être payé de son exil, mais on pourrait dès lors avancer que les artisans de la Révolution tranquille, de fervents lecteurs de Mounier et de Maritain, et personnalistes comme l'ont démontré Meunier et Jean-Philippe Warren dans Sortir de la "Grande noirceur » ${ }^{126}$, allaient lui donner raison.

\section{NOTES}

${ }^{1}$ Hertel est mort dans un hôpital montréalais le 4 octobre 1985 à l'âge de 80 ans, quelques mois à peine après avoir été rapatrié de France.

${ }^{2}$ Gérard Pelletier, « Hertel en ce temps-là (Pages de journal) », L'Incunable, $20^{e}$ année, $\mathrm{n}^{\circ} 1$ (mars 1986,) p. 12. Le texte de Pelletier fait partie de l'important dossier "À la mémoire de François Hertel», publié en mars 1986 par la Bibliothèque nationale du Québec dans son bulletin L'Incunable. Denis Roy et al., L'incunable, $20^{\circ}$ année, $\mathrm{n}^{\circ} 1$ (mars 1986).

${ }^{3}$ Jean Éthier-Blais et Pierre de Grandpré, «François Hertel », dans Pierre de Grandpré, dir., Histoire de la littérature française du Québec, tome II, 1900-1945, Montréal, Librairie Beauchemin, 1968, p. 229.

${ }^{4}$ Pierre Elliott Trudeau, Mémoires politiques, Montréal, Le Jour Éditeur, 1993, p. 32. Ces propos de Trudeau sont rapportés par Claude Corbo, dans La mémoire du cours classique (Outremont, Les Éditions Logiques, 2000, p. 282).

${ }^{5}$ L'expression est d'Alain Pontaut. Elle apparait dans un article intitulé « François Hertel : "Le bilinguisme est un crime" ». L'article a paru dans La Presse, mais on ignore à quelle date, celle donnée par Claude Pelletier dans Francois Hertel : dossier de presse 1938-1985 (Sherbrooke, Bibliothèque du Séminaire de Sherbrooke, 1986, 102 p.) n'étant pas exacte.

${ }^{6}$ Paul Wyczynski explique très bien que Hertel portait en effet plusieurs chapeaux. Paul Wyczynski, «Essai sur la littérature : des origines à 1960 », dans Paul Wyczynski, François Gallays et Sylvain Simard, dir., Archives des lettres canadiennes, tome VI, L'essai et la prose d'idées au Québec, Montréal, Fides, 1985, p. 102.

${ }^{7}$ Guy Sylvestre, «Pour un ordre personnaliste », Le Droit, $31^{\mathrm{c}}$ année, $\mathrm{n}^{\circ} 42(20$ février 1943), p. 5. 
${ }^{8}$ Marie-Joseph d'Anjou, "Pour un ordre personnaliste», L'Action nationale, vol. XXI, avril 1943, p. 339.

'Jean-Claude Simard, "La philosophie française des XIX et XX ${ }^{\mathrm{e}}$ siècles », dans Raymond Klibansky et Josiane Boulad-Ayoub, dir., La pensée philosophique d'expression française au Canada. Le rayonnement du Québec, Sainte-Foy, Les Presses de l'Université Laval, 1998, p. 63. (Coll. "Zêtêsis »).

${ }^{10}$ Fernand Dumont rappelle en effet qu'à l'époque, à peu près tous au Québec estimaient que la crise économique des années trente avait une source morale : "Crise morale et même "métaphysique", traduction de la crise en termes d"honnêteté politique", mépris plus profond de la politique elle-même: voilà les postulats principaux du diagnostic porté sur les années 30 , sur leur situation historique, par les idéologues de l'époque ", affirme-t-il. Fernand Dumont, «Les années 30 : la première Révolution tranquille », dans Fernand Dumont, Jean Hamelin et Jean-Paul Montminy, dir., Idéologies au Canada français (1930-1939), Québec, Presses de l'Université Laval, 1978, p. 5. (Coll. «Histoire et sociologie de la culture »).

${ }^{11}$ En faisant allusion aux détracteurs de Hertel, nous pensons plus précisément à son supérieur, Émile Papillon, et aux critiques qu'il lui adressait à l'été 1942, sur lesquelles nous reviendrons. Par ailleurs, il est connu que Hertel a remis en cause sa foi au début des années cinquante. Il s'explique à ce sujet dans Cité libre, en 1951 : «Plus je vieillis d'ailleurs, plus je me rends compte que je me suis trompé à vingt ans sur mon orientation et que je n'étais nullement fait pour les ordres. [...] Enfin j'ai aussi évolué dans le domaine de la pensée. Je ne suis plus tout à fait aussi sûr de tout ce que j'enseignais (maintenant que j'ai davantage étudié) que je l'étais autrefois » (François Hertel, «Lettre à mes amis ", Cité libre, vol. 2, nº 1 [février 1951], p. 35). Mais la relation de Hertel à la foi et la religion catholique est trop complexe pour être traitée de façon pertinente en quelques lignes. Elle ferait à elle seule l'objet d'un article.

12 Jean-Paul Brodeur, "De l'orthodoxie en philosophie», Philosophiques, vol. III, $\mathrm{n}^{\circ} 2$ (octobre 1976), p. 209. L'auteur se réfère à l'enquête effectuée par le professeur de Sir George Williams, Stanley French, qui révélait que plus de la moitié des professeurs de philosophie se réclamaient encore « des principes du thomisme » au Québec en 1962.

${ }^{13}$ Adélard Dugré, « Apologétique et controverse », L'Académie canadienne SaintThomas d'Aquin, 4, Quatrième session, Québec, L'Action catholique, 1935, p. 25.

${ }^{14}$ Brodeur, loc. cit., p. 211. 
${ }^{15}$ Jean-Paul Brodeur, "À propos d'une question de Fernand Dumont ", dans Philosophie au Québec, Montréal et Paris-Tournai, Bellarmin et Desclée, 1976, p. 65. (Coll. «L'univers de la philosophie »).

${ }^{16}$ Charles de Koninck, De la primauté du bien commun contre les personnalistes. Le principe de l'ordre nouveau, Québec et Montréal, Éditions de l'Université Laval et Éditions Fides, 1943, 195 p. Des extraits de cet essai parurent dans la Semaine religieuse de Québec, $55^{\circ}$ année, $\mathrm{n}^{\text {os }} 12,13,14,15,1942$, signale Roland Houde dans «Jacques et Raïssa Maritain au Québec, II », Relations, $\mathrm{n}^{\circ} 384$ (juilletaoût 1973), p. 216.

17 "[Еterni Patris] Lettre encyclique de Notre très saint Père le pape Léon XIII ", Études religieuses, bistoriques et littéraires, $23^{\mathrm{e}}$ année, septembre 1879, pp. 351-352.

${ }^{18}$ "Les grandes encycliques pontificales de Pie VI à Paul VI : Léon XIII ", dans Michel Dubost, dir., Théo : L'encyclopédie catholique pour tous, Paris, Éditions Droguet-Ardant et Fayard, 1992, p. 552.

${ }^{19}$ Yvan Cloutier, «L'hégémonie philosophique dominicaine dans le Québec des années 1920-1950 : jalons ", Les Cabiers d'bistoire du Québec au XX $X^{e}$ siècle, $\mathrm{n}^{\circ} 4$ (été 1995), p. 27.

${ }^{20}$ Ceslas-Marie Forest, Mémoires, dans Yvan Lamonde et Benoît Lacroix, « Les débuts de la philosophie universitaire à Montréal. Les mémoires du doyen Forest, o.p. (1885-1970) ", Philosophiques, vol. III, no 1 (avril 1976), p. 78. Par ailleurs, $\mathrm{M}^{\mathrm{gt}}$ Pâquet (1859-1942) a également dirigé pendant près de dix ans l'École supérieure de philosophie (1926-1935) de l'Université Laval.

${ }^{21}$ Villeneuve devint archevêque de Québec en décembre 1931, puis cardinal en mars 1933.

${ }^{22}$ L'Académie canadienne Saint-Thomas d'Aquin a été fondée le 16 janvier 1930. Certains disent que Pâquet en fut le fondateur, mais d'après Brodeur, l'initiative revient au cardinal Rouleau. $\mathrm{M}^{\mathrm{gt}} \mathrm{Pâquet}$ en fut le premier président.

${ }^{23}$ Jean-Marie-Rodrigue Villeneuve, « Le rôle de la philosophie dans l'œuvre des universités catholiques ", L'Académie canadienne Saint-Thomas d'Aquin, I, Première session, Québec, L’Action catholique, 1932, p. 213.

${ }^{24}$ Maurice Roy, "Pour l'histoire du thomisme au Canada ", Journées thomistes 1 : essais et bilans, Ottawa, Collège dominicain, 1935, p. 26.

${ }^{25}$ Louis-Marie Régis, « La philosophie au Canada français ", Communauté cbrétienne, vol. 12, $\mathrm{n}^{\circ} 70$ (juillet-août 1973), pp. 264-265. Par ailleurs, le surnom de docteur Angélique donné à Thomas d'Aquin n'a aucun caractère officiel. Tho- 
mas d'Aquin porte également le titre de docteur de l'Église, attribué par l'Église elle-même (dans Théo, pp. 23-24).

${ }^{26} \mathrm{Au}$ long de sa carrière universitaire, Thomas d'Aquin a rédigé deux ouvrages majeurs : Summa contra Gentiles (1259-1264), et Summa theologica (12671274).

${ }^{27}$ E.-Martin Meunier, Les transformations de l'éthique catbolique au XX $X^{e}$ siècle : de la mouvance personnaliste à l'esprit du catbolicisme contemporain. La contribution française, thèse de doctorat (sociologie), Université Laval, 2001, p. 55.

${ }^{28}$ Marie-Dominique Chenu, «Thomas d'Aquin, saint (1224 ou 1225-1274) ", Dictionnaire des philosophes, $2^{\mathrm{e}}$ éd., Paris, Encyclopædia Universalis, Albin Michel, 2001, p. 1514.

${ }^{29}$ Jacqueline Russ, Histoire de la philosophie : de Socrate à Foucault, Paris, Hatier, 1998, p. 54.

${ }^{30}$ Ibid., p. 53. Thomas d'Aquin, par ailleurs, s'est inspiré d'Aristote pour démontrer l'existence de Dieu : "Il y a du mouvement dans le monde. Or tout ce qui est en mouvement est mû par autre chose. Mais il n'est pas possible de remonter à l'infini. Il est donc nécessaire d'en venir à un premier moteur qui ne soit mû par aucun autre. Ce premier moteur, c'est Dieu ", résume en des termes simples Russ (p. 53).

${ }^{31}$ Hermas Bastien, Itinéraires philosophiques, Montréal, Librairie d'Action canadienne-française, 1929, p. 179.

${ }^{32}$ Géry Prouvost, Thomas d'A Auin et les thomismes. Essai sur l'histoire des thomismes, Paris, Les Éditions du Cerf, 1996, p. 9.

${ }^{33}$ Tous quatre Français : Jacques Maritain (1882-1973) et Étienne Gilson (18841978), philosophes laïques ; A. D. Sertillanges (1863-1948) et G. GarrigouLagrange (1877-1964), philosophes et théologiens dominicains.

${ }^{34}$ En 1975, Hertel prétend que son thomisme « ostentatoire » « lui était dicté par la nécessité d'obtenir l'imprimatur», et que Pour un ordre personnaliste « avait été fortement censuré par lui avant de l'être par d'autres, plus pointilleux » (dans Mystère cosmique et condition bumaine, Montréal, Les Éditions La Presse, 1975, p. 15). Nous ne croyons pas toutefois qu'il soit indiqué de tenir compte de cette confession tardive, Hertel spécifiant, une page plus loin, qu'« il ne répudiait absolument pas ses œuvres philosophiques de cette époque», et qu'il croyait « avoir été sincère, quoique parfois naïf, dans toutes les phases de son évolution ». 
${ }^{35}$ Nous nous sommes nous-même inspirée de l'ouvrage du philosohe français Émile Peillaube, Initiation à la philosophie de Saint Thomas. Le manuel, rédigé en français (la plupart l'étaient en latin à l'époque), est paru dans les années où Hertel faisait sa philosophie. Peillaube fut l'un des fondateurs et le premier doyen de la Faculté de philosophie de l'Institut catholique de Paris, où l'étude de saint Thomas tenait la première place. Sur le plan doctrinal, on ne lui attribuait aucune audace susceptible de contrarier les contemporains de Hertel au Canada français, à qui l'on n'aurait su reprocher des écarts en la matière. Enfin, Peillaube a soutenu Maritain au début de sa carrière, l'ayant aidé à obtenir un poste à l'université.

${ }^{36}$ Hertel est bien près de semer la confusion dans l'esprit du lecteur : il élabore une métaphysique du travail non pas selon le sens que ce mot a de nos jours, mais selon la définition qu'en donnaient les scolastiques. Pour mémoire, ceux-ci considéraient que le travail était du domaine de l'art au sens le plus universel du terme ; Maritain l'explique longuement dans Art et scolastique. Mais Hertel ne prévient pas le lecteur d'un tel crochet par la scolastique, pas plus qu'il ne prend la peine d'expliquer la rupture qui s'est produite entre les beaux-arts et les métiers - que l'on retrouve dans la langue à partir de 1762, note Maritain. Jacques Maritain, « Art et scolastique », dans Jacques et Räissa Maritain, CEuvres complètes I, Fribourg et Paris, Éditions universitaires et Éditions Saint-Paul, 1986, p. 619.

${ }^{37}$ Par opposition à l'âme végétative et à l'âme sensitive.

${ }^{38}$ François Hertel, Pour un ordre personnaliste, Montréal, Les Éditions de l'Arbre, 1942, p. 330.

${ }^{39}$ Ibid., p. 94.

${ }^{40}$ Meunier, op. cit., p. 24. Par ailleurs, lors d'une conférence prononcée à l'Université Fordham, à New York en octobre 2003, le cardinal Avery Dulles déclarait que la philosophie personnaliste du pape Jean-Paul II l'avait influencé dans ses diverses prises de position en matière de théologie et de doctrine sociale. «Depuis son accession au trône de saint Pierre, Jean-Paul II a revu l'essentiel de la tradition catholique dans une perspective personnaliste. [...] Il entérine vigoureusement tous les dogmes de l'Église, mais il les envisage sous un angle personnaliste ", a-t-il affirmé (Tracy Early, " Cardinal says pope's personalism a distinctive approach ", Catbolic News Service, 22 octobre 2003). Le texte de la conférence du Père Dulles a été publié en février 2004 dans la revue America. "John Paul II and The Mystery of The Human Person", America, vol. 190, n 3 (2 février 2004). 
${ }^{41}$ Jean-Louis Loubet Del Bayle, «Le mouvement personnaliste français des années 1930 et sa postérité ", Politique et sociétés, vol. 17, nos 1-2 (1998), p. 219.

${ }^{42}$ Emmanuel Mounier, «Manifeste au service du personnalisme », Esprit, $5^{\mathrm{c}}$ année, $\mathrm{n}^{\circ} 49$ ( $1^{\text {er }}$ octobre 1936), p. 7. Mounier publiait en 1935 Révolution personnaliste et communautaire.

${ }^{43}$ Jean-Marie Grevillot, Les grands courants de la pensée contemporaine, Paris, Édition du Vitrail, 1948, p. 171.

${ }^{44}$ Mounier, loc. cit., p. 7.

${ }^{45}$ Ibid., p. 8.

${ }^{46}$ Jacques Maritain, «La personne et le bien commun », Revue thomiste, $\mathrm{LIV}^{\mathrm{e}}$ année, tome LXVI, no 2 (mai-août 1946), pp. 237-278. Christian Roy relie le développement du personnalisme en France à deux groupes, celui à l'origine de la revue L'Ordre nouveau (1933) et celui de la revue Esprit (1932). « Le premier, d'inspiration nietzschéenne, devait se perpétuer après la guerre dans le mouvement fédéraliste européen ; le second, d'inspiration chrétienne, devait se prolonger dans le progressisme catholique. »Christian Roy, « De La Relève à Cité libre : avatars du personnalisme au Québec », Vice Versa, n 17 (décembre 1986-janvier 1987), p. 14.

${ }^{47}$ François Hertel, Leur inquiétude, Montréal, Éditions « Jeunesse » A.C.J.C. et Éditions Albert Lévesque, 1936, p. 36.

${ }^{48}$ Hertel, Pour un ordre personnaliste, op. cit., p. 93.

${ }^{49}$ Lettre de François Hertel à Guy Sylvestre, janvier 1943, Bibliothèque nationale du Canada (Ottawa), Collection des manuscrits littéraires, Fonds GuySylvestre LMS-0110, Correspondance, FO-HO, boîte n 4, François Hertel.

${ }^{50}$ Ibid.

${ }^{51}$ Jacques Maritain, Humanisme intégral, Paris et Montréal, Fernand Aubier et Éditions de l'Arbre, 1939 [1936], p. 84.

${ }^{52}$ Maritain, «La personne et le bien commun », loc. cit., p. 238. Les propos de Maritain sont toutefois antérieurs à la parution du texte, remontant, explique l'auteur dans une note de bas de page, à des conférences prononcées en 1939 et en 1945 respectivement, et à d'autres études antérieures.

${ }^{53}$ Meunier, op. cit., p. 144.

${ }^{54}$ Lettre de Hertel à Sylvestre, op. cit. 
${ }^{55}$ Hertel y fait allusion dans Leur inquiétude (p. 102), évoquant les « conférences enthousiastes " du " grand philosophe », à Montréal en octobre 1934. On retrouve les textes de ces conférences dans Le Devoir: 16 octobre 1934,pp. 2 et $3 ; 19$ octobre 1934 , p. $6 ; 22$ octobre 1934, p. $8 ; 23$ octobre 1934, p. $8 ; 24$ octobre 1934, p. 10.

${ }^{56}$ Yvan Cloutier, "Maritain, Mounier et le Québec : une tutelle éditoriale », dans Claude Hauser et Yvan Lamonde, dir., Regards croisés entre le Jura, la Suisse romande et le Québec, Québec et Porrentruy (Suisse), Presses de l'Université Laval et Office du patrimoine et de la culture de la République et Canton du Jura, 2002, p. 227.

${ }^{57}$ Ibid., p. 224.

${ }^{58}$ Ibid., p. 227.

${ }^{59}$ Lionel Groulx, « Primauté du spirituel, par Jacques Maritain », L'Action française, vol. XVIII, $\mathrm{n}^{\circ} 3$ (septembre 1927), p. 188.

${ }^{60}$ Bastien, op. cit., p. 136.

${ }^{61}$ Outre Mounier et Maritain, d'autres intellectuels, faut-il rappeler, ont contribué au personnalisme : notamment les animateurs du mouvement l'Ordre nouveau et les collaborateurs de la revue du même nom, en France, les Alexandre Marc, Arnaud Dandieu, Nicolas Berdiaeff, Robert Aron, DanielRops. Hertel les a certainement lus, mentionnant tous ces derniers (à l'exception de Marc) dans Leur inquiétude et Pour un ordre personnaliste. Nous nous sommes arrêtée à Maritain parce qu'il est de loin le plus cité (à onze reprises dans Pour un ordre personnaliste et à quatre reprises dans Leur inquiétude); Hertel l'a identifié comme son maitre, et il reprend mot pour mot, établirons-nous, la définition que donne le philosophe français d'une civilisation personnaliste.

${ }^{62}$ Hertel fait allusion à Humanisme intégral dans Leur inquiétude, mais dans une moindre mesure, en trois occasions seulement.

${ }^{63}$ Se reporter au chapitre intitulé «Primat de la contemplation", dans Primauté du spirituel, Paris, Plon, 1927, pp. 166-179.

${ }^{64}$ Hertel se démarque cependant de Maritain sur certains points. Quelques différences entre Humanisme intégral et Pour un ordre personnaliste valent d'être relevées ; elle résident notamment dans le vocabulaire, le choix des mots, des idées présentes chez l'un, absentes chez l'autre. Par exemple, Hertel parle de justice et de charité, Maritain, de vérité, de justice et d'amour ; Maritain parle de démocratie personnaliste (tout en précisant qu'il ne concevait pas la démocratie à la manière de Jean-Jacques Rousseau), ce à quoi Hertel ne se hasarde 
pas. Sur le plan des idées, Maritain s'attache à développer la notion de pluralisme, ce que Hertel ne fait pas, sans doute parce que la société à laquelle il appartenait était encore très homogène. Hertel se contente d'affirmer que le corporatisme chrétien correspond à la cité pluraliste de Maritain (Pour un ordre personnaliste, p. 114), et que dans un État corporatiste, le libéralisme économique aurait laissé place « à la cité pluraliste, à la cité vraiment sociale » (p. 132). Par ailleurs, Maritain dénonce plus vigoureusement le totalitarisme d'État, celui qu'on observe en Italie tout autant que les autres.

${ }^{65}$ Simard, loc. cit., p. 58.

${ }^{60}$ Roger Benjamin, Notion de personne et personnalisme chrétien, Paris et La Haye, Mouton Éditeur, 1972 [1971], pp. 111-112.

${ }^{67} \mathrm{La}$ distinction entre individu et personne serait néanmoins antérieure à Maritain, de l'avis de Benjamin (Ibid., p. 112) : elle s'ébauchait déjà sous la plume du père Garrigou-Lagrange, dès 1908.

${ }^{68}$ Jacques Maritain, Trois réformateurs, Paris, Librairie Plon, 1925, pp. 30-31. (Coll. « Le roseau d'or »).

${ }^{69}$ Simard, loc. cit., p. 58.

${ }^{70}$ Benjamin, op. cit., p. 114.

${ }^{71}$ Jean-Louis Loubet Del Bayle, Les non-conformistes des années 30 : une tentative de renouvellement de la pensée politique franşaise, Paris, Éditions du Seuil, 1969, p. 343.

${ }^{72}$ Maritain, Trois réformateurs, op. cit., pp. 31-32.

${ }^{73}$ Hertel, Pour un ordre personnaliste, op. cit., p. 95.

${ }^{74}$ Ibid., p. 95.

${ }^{75}$ Ibid., p. 99.

${ }^{76}$ Maritain, Trois réformateurs, op. cit., pp. 30-31.

${ }^{77}$ Roy, loc. cit., p. 15.

${ }^{78}$ Hertel, Pour un ordre personnaliste, op. cit., pp. 8-9.

${ }^{79}$ Jacques Croteau, Les fondements thomistes du personnalisme de Maritain, Ottawa, Éditions de l'Université d'Ottawa, 1955, p. 41.

${ }^{80}$ Lettre de Hertel à Sylvestre, op. cit. 
${ }^{81}$ Ce Cours de philosophie, publié d'abord en latin, fut traduit en français. Il compte dans cette langue deux volumes : Cours de philosophie I : logique, métaphysique, philosophie de la nature, Québec, édité par l'auteur, 1942, 410 p. ; Cours de philosophie II : monastique ou étbique, économique, politique, Québec, édité par l'auteur, $1942,469 \mathrm{p}$.

${ }^{82}$ Hertel, Pour un ordre personnaliste, op. cit., p. 108.

${ }^{83}$ Grenier, Cours de philosophie II, op. cit., p. 346.

${ }^{84}$ Hertel, Pour un ordre personnaliste, op. cit., p. 115.

${ }^{85}$ Grenier, Cours de philosophie II, op. cit., p. 346.

${ }^{86}$ Ibid., pp. 347-348.

${ }^{87}$ Ibid., p. 346.

${ }^{88}$ Louis Lachance, L'bumanisme politique de saint Thomas, tome 1, Paris et Ottawa, Recueil Sirey et Éditions du Lévrier, 1939, p. 10. Dans l'édition définitive, datant de 1965, Lachance spécifie que Lapersonne et le bien commun (1946), de Maritain, est au nombre des études qui ne lui semblent pas répondre aux préoccupations exprimées dans son propre essai. Louis Lachance, L'bumanisme politique de saint Thomas d'Aquin, Paris et Montréal, Éditions Sirey et Éditions du Lévrier, 1965, p. 11.

${ }^{89}$ Jean-Claude Simard affirme que la parution de Pour un ordre personnaliste « a déclenché une controverse mémorable avec Charles de Koninck » (dans " Découvrons la philosophie avec François Hertel », Philosophiques, vol. 26, $\mathrm{n}^{\circ} 2$ [automne 1999], p. 374). Il aurait été intéressant que M. Simard donne plus de précisions à ce sujet, car les revues spécialisées de l'époque, telles La Nouvelle Relève, Culture, Revue dominicaine, Carnets viatoriens, La semaine religieuse, ne font pas état de cette controverse. Leur critique de Pour un ordre personnaliste est même favorable dans l'ensemble. Croteau, pour sa part, fait mention d'un débat dans les revues The Modern Schoolman, Medieval Studies et Laval théologique etphilosophique. Or ledit débat n'oppose pas Hertel et de Koninck, mais ce dernier et le dominicain I. Th. Eschmann. Voir The Modern Schoolman, vol. XXII, $\mathrm{n}^{\circ} 4$ (mai 1945), pp. 183-208, et Laval théologique et philosophique, vol. I, $\mathrm{n}^{\circ} 2$ (1945), pp. 9-109. Par ailleurs, l'article de Maritain, «La conquête de la liberté », paru dans Gants du ciel en septembre 1943, ne peut être considéré comme pièce justificative, car Maritain soutient que l'article avait été écrit bien avant la sortie du livre de de Koninck. Voir Lettre de Jacques Maritain à Guy Sylvestre, 28 juin 1943, dans « Lettres : Jacques et Raïssa Maritain », p. 105. En fait, il faut lire les recensions du livre de de Koninck, plutôt que de Pour un 
ordre personnaliste, pour saisir que le personnalisme suscite la controverse au Canada français.

${ }^{90}$ Frédéric Saintonge, «Livres récents. Philosophie et essais. Charles de Koninck: De la primauté du bien commun (contre les personnalistes); le principe de l'ordre nowveau », Relations, $\mathrm{III}^{\mathrm{c}}$ année, $\mathrm{n}^{\circ} 33$ (septembre 1943), p. 249.

${ }^{91}$ Croteau, op. cit., p. 43.

${ }^{92}$ Jean-Marie-Rodrigue Villeneuve, «Préface », dans de Koninck, op. cit., p. xi. ${ }^{93}$ Eugène Babin, « Les livres canadiens. De Koninck, Charles, De la primauté du bien commun contre lespersonnalistes. Le principe de l'ordre nouveau ", Culture, vol. IV, $\mathrm{n}^{\circ} 3$ (septembre 1943), p. 430.

${ }^{94}$ Ibid., p. 432.

${ }^{95}$ Cloutier, loc. cit., p. 231.

${ }^{96}$ Yves Simon, "On the Common Good», The Review of Politics, vol. 6, $\mathrm{n}^{\circ} 4$ (octobre 1944), pp. 532-533. L'extrait reproduit est une traduction libre.

${ }^{97}$ Cloutier a tiré cette information de la thèse de Bérangère Bois, Jacques Maritain : controverses et influences 1930-1960, thèse de maîtrise (histoire contemporaine), Université Jean-Moulin / Lyon 3, 1995-1996, pp. 158-161, cité dans Cloutier, loc. cit., p. 231.

${ }^{98}$ Babin, loc. cit., p. 432.

${ }^{99}$ Hertel, Leur Inquiétude, op. cit., p. 36.

${ }^{100}$ Jean-Marie Mayeur et al., Histoire du christianisme des origines à nos jours, tome XII, guerres mondiales et totalitarismes (1914-1958), Paris, Desclée-Fayard, 1990, p. 167.

${ }^{101}$ Bastien, op. cit., p. 159.

${ }^{102}$ Forest, loc. cit., p. 78.

${ }^{103}$ Yvan Lamonde, Territoires de la culture québécoise, Sainte-Foy, Presses de l'Université Laval, 1991, p. 221.

${ }^{104}$ Jean Éthier-Blais, Le seuil des vingt ans, Montréal, Leméac, 1992, pp. $187-$ 188.

${ }^{105}$ François Hertel, Nous ferons l'avenir, Montréal, Fides, 1945, pp. 40-41. 
${ }^{106}$ Lettre d'Émile Papillon, s.j., à Rodolphe Dubé [François Hertel], 18 juillet 1942, Bibliothèque nationale du Québec (Montréal), Fonds François-Hertel, MSS - 385 .

${ }^{107}$ Paul Toupin, De face et de profil, Montréal, Éditions Pierre Tisseyre, 1977, p. 37.

${ }^{108}$ Samuel Baillargeon, Littérature canadienne-franşaise, Montréal, Fides, $2^{\mathrm{e}}$ édition, 1957, pp. 391-392.

${ }^{109}$ Hertel donna quelques cours sur l'histoire de la philosophie, mais l'expérience prit fin moins de deux ans après avoir commencé. Voir Jean Tétreau, Hertel, l'homme et l'auvre, Montréal, Pierre Tisseyre, 1986, p. 128.

${ }^{110}$ Ibid., p. 128.

${ }^{111}$ Pontaut, loc. cit. En 1950, Hertel avait fait le même constat, dans une lettre à Roger Duhamel : «Je suis actuellement boycotté par les libraires canadiens et, disons-le, par les curés. Le boycottage est sourd, mais sûr. Cela entrave la vente et m'embarrasse ». Lettre de François Hertel à Roger Duhamel, juin 1950, Centre de recherche Lionel-Groulx (Outremont), Fonds Roger-Duhamel, P46 / D1, 183.

${ }^{112}$ Yolande Grisé, «François Hertel, l'enfant terrible des lettres québécoises, n'est plus », Lettres québécoises, $\mathrm{n}^{\circ} 40$ (hiver 1985-86), p. 8.

${ }^{113}$ Roberval Paradis, «Pour un ordre personnaliste », Le Quartier latin, vol. XXVI, $\mathrm{n}^{\circ} 3$ (22 octobre 1943), p. 6. Dans son commentaire, l'étudiant avoue ne pas partager « l'enthousiasme béat » qui voudrait, selon lui, faire de Pour un ordre personnaliste « un second évangile ». Il estime que l'essai de Hertel ne comporte « rien de bien neuf » qu'il n'ait déjà appris.

${ }^{114}$ Jean Éthier-Blais, " Exils ", Littérature canadienne-française : Conférences J. A. de Sève, Montréal, Presses de l'Université de Montréal, 1969, p. 129.

${ }^{115}$ Maritain a dénoncé, « d'un point de vue théologique, le mythe de la Guerre sainte ». Or les évêques espagnols (sauf trois d'entre eux) ont soutenu sans réserve, dès 1937, le général Franco (dans Théo, p. 1143).

${ }^{116}$ Louis-Philippe Roy, "À travers le désastre ", L'Action catholique, 24 mars 1941, p. 4. Roy précise, dans le même article, que « L'Action catholique a toujours préféré le jugement de l'épiscopat espagnol sur la légitimité et l'opportunité de la révolution franquiste, si tant est qu'on puisse appeler révolution ce mouvement libérateur $"$. 
${ }^{117}$ Hertel, Leur inquiétude, op. cit., p. 155. Ces propos, faut-il préciser, ont été supprimés de l'édition définitive. Mais en 1944, la guerre achevait et Hertel avait déjà quitté les Jésuites.

${ }^{118}$ Jacques Maritain, $A$ travers le désastre, New York, Éditions de la Maison française, 1941, pp. 77-78. (Coll. « Voix de France »).

${ }^{119}$ Jean-Marie-Rodrigue Villeneuve, " Texte de l'allocution de Son Excellence », Le Devoir, 10 février 1941, p. 6.

${ }^{120}$ François Hertel, "Cette Europe à reconstruire », L'Action nationale, $11^{\mathrm{e}}$ année, vol. XXI, février 1943, p. 128.

${ }^{121}$ Jacques Maritain, « Le cas de M. Jacques Maritain », dans Jacques et Raïssa Maritain, Euvres complètes VII, Fribourg et Paris, Éditions universitaires et Éditions Saint-Paul, 1988, pp. 1236-1237.

${ }^{122}$ Albert Jamet, « M. Maritain. Un penseur ? Oui. Mais un chef ? ", Le Devoir, 15 mai 1943, pp. 1-2. Le Devoir publiera une réplique de Maritain dans l'édition du 26 mai, à côté d'une riposte de Dom Jamet.

${ }^{123}$ Ibid., p. 1.

${ }^{124}$ Jean Le Moyne, "Les Maritain - de loin, de près ", Écrits du Canada français, $\mathrm{n}^{\circ} 49$ (1983), pp. 61-62.

${ }^{125}$ Éric Amyot, Le Québec entre Pétain et De Gaulle : Vichy, la France libre et les Canadiens français, 1940-1945, Montréal, Fides, 1999, pp. 318-319.

${ }^{126}$ E.-Martin Meunier et Jean-Philippe Warren, Sortir de la " Grande noirceur » : l'horizon "personnaliste » de la Révolution tranquille, Montréal, Éditions du Septentrion, 2002, $210 \mathrm{p}$. 